\title{
Monitoring mortality as an indicator of influenza in Catalonia, Spain
}

\author{
Àngela Domínguez, Pilar Muñoz, Anna Martínez, Angels Orcau
}

\begin{abstract}
Study objective - This study aimed to investigate the behaviour of two indicators of influenza activity in the area of Barcelona and to evaluate the usefulness of modelling them to improve the detection of influenza epidemics.

Design - Descriptive time series study using the number of deaths due to all causes registered by funeral services and reported cases of influenza-like illness. The study concentrated on five influenza seasons, from week 45 of 1988 to week 44 of 1993 . The weekly number of deaths and cases of influenza-like illness registered were processed using identification of a time series ARIMA model.
\end{abstract}

Setting - Six large towns in the Barcelona province which have more than 60000 inhabitants and funeral services in all of them.

Main results - For mortality, the proposed model was an autoregressive one of order 2 (ARIMA $(2,0,0)$ ) and for morbidity it was one of order 3 (ARIMA $(3,0,0)$ ). Finally, the two time series were analysed together to facilitate the detection of possible implications between them. The joint study of the two series shows that the mortality series can be modelled separately from the reported morbidity series, but the morbidity series is influenced as much by the number of previous cases of influenza reported as by the previous mortality registered.

Conclusions - The model based on general mortality is useful for detecting epidemic activity of influenza. However, because there is not an absolute gold standard that allows definition of the beginning of the epidemic, the final decision of when it is considered an epidemic and control measures recommended should be taken after evaluating all the indicators included in the influenza surveillance programme.

( $(\mathcal{F}$ Epidemiol Community Health 1996;50:293-298)

Until the 1950s the concept of surveillance was confined to observation of healthy people who had been in contact with individuals who were seriously ill. Its aim was to detect, as early as possible, the appearance of signs and symptoms of illness and to act accordingly. It was in the $60 \mathrm{~s}$ that the concept of epidemiological surveillance emerged. This, as first described by Langmuir, ${ }^{1}$ involves the "continued watchfulness over the distribution and trends of in- cidence through the systematic collection, consolidation and evaluation of morbidity and mortality reports and other relevant data. Intrinsic in the concept is the regular dissemination of the basic data and interpretations to all who have contributed and to all others who need to know."

In recent years some authors have begun to use the term "public health surveillance" in an attempt to both apply the concept not just to illnesses but to any phenomenon of interest to health administrators, and to tie up surveillance with the adoption of health measures for controlling problems. ${ }^{23}$ The aims of public health surveillance are to describe the pattern of illnesses, to detect epidemics, to know about isolated cases of rare diseases, to identify risk factors, to evaluate prevention and control programmes, and to project the future health needs of the population. ${ }^{4}$ Of all these aims, that of detecting epidemic situations continues to be a challenge for the different public health services, especially when it is a question of monitoring illnesses which, like influenza, have an epidemic behaviour that is not well known. ${ }^{5}$ The main feature of the influenza virus is that it periodically changes its antigenic structure, which usually means a rapid spread of the virus and the consequent appearance of excesses in morbidity and mortality, since the population's immunity to the new variant of virus is very low or nil. ${ }^{6}$ International health organisations agree on the necessity of organising influenza surveillance systems which suit the resources and requirements of each country. ${ }^{78}$ Moreover, such international cooperation guarantees the availability of circulating strains each season, thus making it possible to adapt the composition of the vaccine to the changes in the influenza virus.

In Spain, there are influenza surveillance programmes in some regions but there is not a global one. In the autonomous community of Madrid, a surveillance system is working with morbidity but not mortality data. Its main aim is to isolate and characterise the circulating influenza virus. ${ }^{9}$ A previous system of monitoring deaths in the city of Barcelona has been shown to be useful in detecting a suspected epidemic of influenza by analysing the mortality data as a univariate time series. ${ }^{10}$

An influenza surveillance programme has been operating in Catalonia since 1988. Its main aim is the identification of the types and subtypes of the causal virus and the early detection of influenza epidemics in order to adopt the adequate control measures: administration of vaccines and antiviral drugs and organisation of the medical services. The 


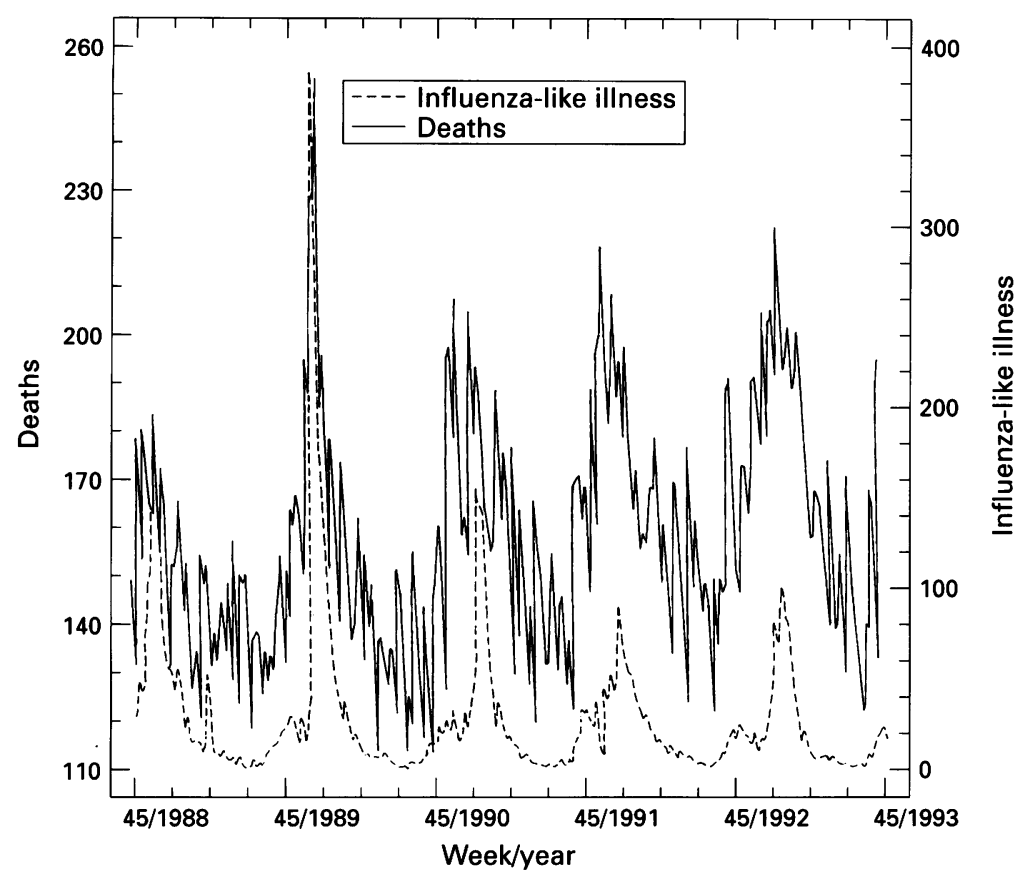

Figure 1 Number of weekly deaths and weekly influenza-like illness reported in the area of Barcelona, week 45, 1988 to week 44, 1993.

programme is based on the systematic collection and evaluation of different indicators during the active influenza season: reported influenza-like illness, mortality from all causes, home visits by general practitioners and paediatricians, and isolations of influenza viruses. Of these indicators, only the first two are available all year round, while the rest are gathered only between weeks 45 and 16, when the influenza monitoring system is functioning actively. We aimed to analyse the behaviour of the number of deaths and the influenza-like illnesses reported in the Barcelona area during various influenza seasons and to evaluate the usefulness of modelling them to improve the detection of influenza epidemics.

\section{Methods}

TIME AMBIT OF THE STUDY

The study concentrated on five influenza seasons, covering the time between week 45 of 1988 and week 44 of 1993 . Epidemic activity has been considered present when an influenza virus has been isolated and more than one of the morbidity indicators included in the system has increased (reported influenza-like illnesses, home visits by general practitioners, or home visits by paediatricians).

\section{SETTING}

Six large towns in the Barcelona province: l'Hospitalet de Llobregat, Mataró, Terrassa, Manresa, Santa Coloma de Gramenet, and Badalona. All these cities have more than 60000 inhabitants and there are funeral services in all of them. According to the 1991 census, ${ }^{11}$ the total population of the towns included in this study was 950334 (16\% of the whole population of Catalonia).

\section{MORBIDITY}

In Catalonia, which is an autonomous community situated in the north east of Spain, all local health chiefs have to report each week the number of cases of certain illnesses, including influenza, on a specific form according to the clinical feature. According to the notification system in Catalonia, suspicion is sufficient for notification. ${ }^{12}$ Given the scant clinical specificity of influenza and the difficulty of laboratory diagnosis, what is really being reported is the amount of influenza-like illness. Weekly numbers of cases of influenza-like illness reported were gathered in the six towns above mentioned.

\section{MORTALITY}

The number of deaths due to all causes registered by the funeral services of the abovementioned townships was used as an indicator of general mortality. A previous study carried out in Barcelona had shown the validity of the information provided by the funeral services. ${ }^{10}$ The Barcelona Territorial Delegation has this information for administrative purposes on a daily basis and thus possesses information ahead of that provided by the Statistical Bulletin of Deaths, which serves as the basis for the official mortality register in our country. The information obtained daily from the towns included in the study has been aggregated into a weekly figures so as to use the same time intervals as the morbidity data.

\section{MODELLING OF THE SERIES}

The Box-Jenkins model was used to construct the indicators. ${ }^{13}$ This involves the processing of time series using identification of the time series ARIMA model, calculation of the parameters laid down in the identification, verification of the model and, once verification has been deemed satisfactory, prediction. ${ }^{1415}$

Verification of the model was carried out with a twofold aim in mind. Firstly, we verified that the estimated parameters were significant using the Student's test for each of the parameters. Next, we determined whether analysis of the residuals was satisfactory. This was done by graphic representation and inspection of the plot of standardised residuals and their autocorrelation function and partial autocorrelation function, in order to confirm that the residuals were "white noise". Finally, a significant reduction in the variation of the original series was confirmed. An anomalous cause was detected using adapted prediction (prediction that is updated week by week) of the number of deaths or notifications of influenza in the following week, as well as confidence intervals. Once the number of real cases was known, if this was above the upper limit of the confidence interval, estimated at $95 \%$ for the prediction, and this situation was maintained for two weeks or more, it was considered a symptom of a possible anomaly of general mortality or influenza morbidity.

These periods were considered high risk periods and in them the predictions and their confidence intervals were not adapted (predictions and confidence intervals were cal- 
Table 1 Interventions carried out in the mortality model

\begin{tabular}{llll}
\hline Weeks & Year & Type of intervention & Concomitant factor \\
\hline 2 to 7 & 1990 & $\mathrm{D}_{\mathrm{a}}$ & Influenza epidemic \\
51 to 2 and 6 to 10 & $1990-91$ & $\mathrm{D}_{\mathrm{b}}$ & No identified \\
51 to 2 and 5 & $1991-92$ & $\mathrm{D}_{\mathrm{b}}$ & No identified \\
4 to 10 & 1993 & $\mathrm{D}_{\mathrm{d}}$ & Influenza epidemic \\
\hline
\end{tabular}

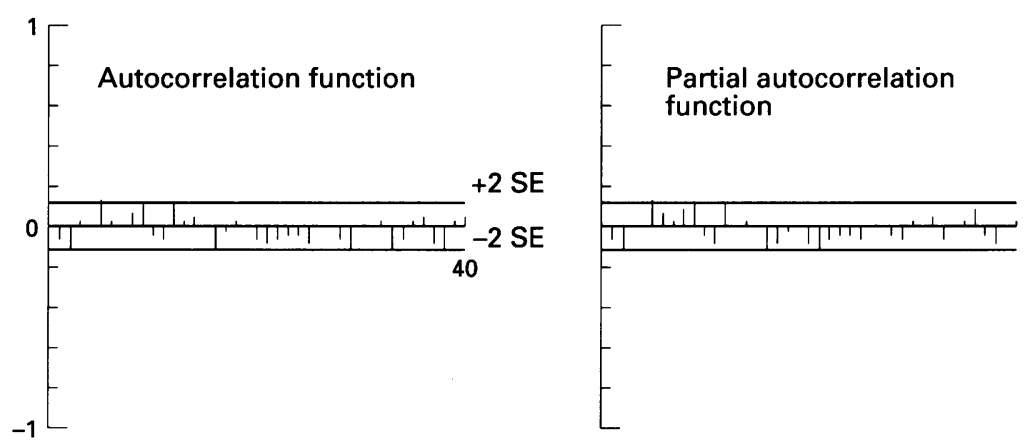

Figure 2 Autocorrelation function and partial autocorrelation function of the mortality series residuals, week 45, 1988 to week 44, 1993.

Table 2 Interventions carried out in the morbidity model

\begin{tabular}{llll}
\hline Weeks & Year & Type of intervention & Concomitant factor \\
\hline 53 to 3 & $1988-89$ & $\mathrm{G}_{\mathrm{a}}$ & Influenza epidemic \\
3 to 7 & 1990 & $\mathrm{G}_{\mathrm{b}}$ & Influenza epidemic \\
7 to 10 & 1991 & $\mathrm{G}_{\mathrm{c}}$ & Other respiratory \\
& & & \\
\hline
\end{tabular}

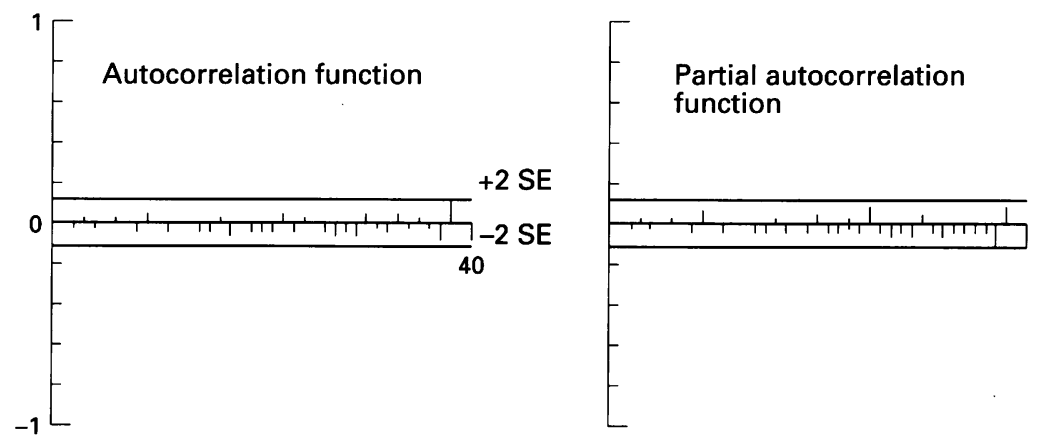

Figure 3 Autocorrelation function and partial autocorrelation function of the morbidity series residuals, week 45, 1988 to week 44, 1993.

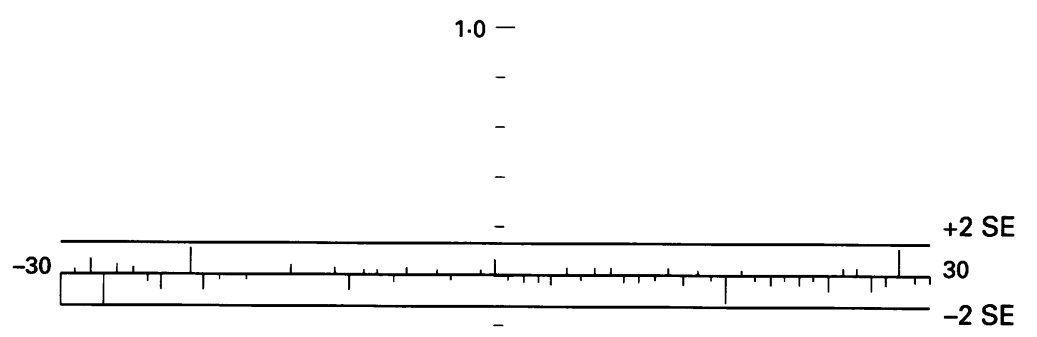

$-1.0-$

Figure 4 Cross correlations of the residuals of mortality at time (t) and morbidity at time $(\mathrm{t}+\mathrm{h})$, week 45, 1988 to week 44, 1993

culated with data that did not take into account observations corresponding to high risk periods) until the number of observations returned to the usual levels.
The choice of models for the time series was made on the basis of their greater sensitivity in detecting anomalous values in short term predictions.

The two time series were analysed together by means of vector autoregressive processes $\operatorname{VAR}(p)^{1617}$ to facilitate the detection of possible implications between the general mortality series and that of influenza-like illness notifications.

\section{Results}

Three seasons during the study period showed epidemic activity. The joint representation of the mortality series and reported morbidity series is to be found in figure 1 , in which the number of weekly influenza notifications has been divided by 20 ; the scale on the left refers to the number of deaths, while the one on the right indicates the number of influenza cases reported. When the number of deaths or the number of reported influenza-like illnesses was abnormally increased, an intervention analysis was done; this consists of adding dummy variables to the ARIMA model, taking the value 1 in the abnormal observations and 0 in the other cases. The intervention analysis was done retrospectively and showed its efficacy because the residual variance diminished and then the predictions were more accurate.

The model proposed for the mortality series is an autoregressive one of order 2 ARIMA $(2,0,0)$, whose description is to be found in the Appendix. Interventions carried out are to be seen in table 1. Two of them agree with the influenza activity detected by virus isolations and other indicators, the third could be explained by an increase in other respiratory infections. The fourth could not be related to any factor; although there were some influenza virus isolations, other indicators did not reveal epidemic activity. In only one period (1988-89) was the intervention not significant despite epidemic activity. The analysis of the residuals was satisfactory. The graph of their functions of autocorrelation and partial correlation can be seen in figure 2 . The variance of residuals was lower than that of the original series $252 \cdot 41$ compared with $599 \cdot 76$.

The model proposed for the reported mobidity series is an autoregressive one of order 3 ARIMA $(3,0,0)$, whose description is to be found in the Appendix. The interventions carried out are in table 2 . Two of these interventions agree with influenza epidemics and the third was explained by an increase of other respiratory infections. The functions of autocorrelation and partial autocorrelation of residuals confirmed that they were white noise, shown in figure 3 . In this case also, the variance of the residuals was lower than that of the original series - $133905 \cdot 29$ compared with 794879-23.

Finally, the time series of mortality and reported morbidity were analysed jointly in order to detect the possible implications between the two, by means of vector autoregressive processes $\operatorname{VAR}(p)$. The joint study of both 
series has shown that the mortality series can be modelled separately from the reported morbidity series, but the morbidity series is influenced as much by the number of cases registered in this series in the previous weeks as by the number of deaths that have occurred in the previous weeks. The results of this model are to be seen in the Appendix. Figure 4 shows the sample cross-correlation of the two sets of residuals.

\section{Discussion}

This study presents models developed to evaluate influenza activity through the number of deaths registered for all causes and the number of reported influenza-like illnesses.

Choi and Thacker, ${ }^{1415}$ in 1981 , were the first to use the ARIMA methods to model pneumonia and influenza mortality in order to estimate the excess deaths attributable to these diseases. The advantage of ARIMA models compared with other statistical techniques is a more accurate prediction. Stroup et al ${ }^{18} \mathrm{com}-$ pared univariate and multivariate models for influenza and all causes of mortality using data from age groups for earlier detection of influenza epidemics. General mortality was also used by some authors as the indicator of influenza activity rather than specific mortality for influenza and pneumonia because the former probably reflects the excess mortality caused by influenza better than the latter. ${ }^{19}{ }^{20}$ Influenza usually affects people with other disorders and not infrequently the death certificate gives these pathologies as the primary cause of death. In a Dutch study, Sprenger et $a l^{20}$ noted that for every officially registered influenza death there were 2.6 deaths for the same cause that were not registered as such. Lui $^{21}$ points out that definitive diagnosis of influenza belongs to the laboratory, and for determining the cause of death these data are not available. In practice, therefore, the decision to classify a death within the category of pneumonia-influenza is subjective and could be biased. Thus, the total number of deaths is an objective datum which could be a better representation of the excess of mortality by influenza.

When only mortality in those over 60 or over 65 was used to predict epidemics, results were usually no better than for general mortality. ${ }^{1819}$ Our results support the idea that general mortality is useful for identifying an increase in influenza activity. The series in this study show that in the periods when an increase in general mortality has occurred, the number of reported influenza-like illnesses had also increased.

The number of reported influenza-like illnesses presents problems of validity - laboratory diagnosis that excludes other acute respiratory affections is not available because of practical difficulties. Glezen, ${ }^{22}$ in the United States, showed that morbidity data are necessary to confirm epidemic activity and that there is a considerable time overlap of influenza and acute respiratory infections of other aetiologies: Mycoplasma pneumoniae, the syncytial respiratory virus, and other respiratory viruses have frequently been identified in cases labelled influenza-like illness. ${ }^{2324}$ Influenza cases may also be wrongly labelled as acute respiratory infections. The problems of lack of predictive value and sensitivity of the reported influenzalike illnesses are a limitation of this indicator and have already been pointed out by other authors. In a study in Tecumseh (Michigan, United States), Sullivan et $a l^{25}$ comment that the target symptoms of influenza vary with age and that it is advisable to determine the aetiology of acute respiratory processes in order to assess adequately the impact of influenza on the community. Fleming and Cross, ${ }^{19}$ also in the United States, reach similar conclusions, showing that the incorrect attribution of respiratory disease epidemics to the influenza virus is a considerable problem when evaluating the efficacy of influenza programmes. Some authors propose the use of acute respiratory dieases as the indicator of morbidity for monitoring influenza. ${ }^{2526}$ Specific studies must be carried out to achieve a deeper knowledge about this point.

The limited value of notified influenza-like illnesses in the early detection of epidemics could also be due to undernotification. ${ }^{27} \mathrm{We}$ do not have any studies that quantify undernotification of influenza, but these have been carried out in other communities and it has been shown that only one tenth of real cases of influenza are declared. ${ }^{28}$ When Sprenger et $a l^{29}$ observe that mortality increases proportionally more than morbidity during influenza epidemics, they attribute this to undernotification. Fleming and Ayres ${ }^{30}$ propose that the level of morbidity required to define an influenza epidemic is a rate of 400 per $10^{5}$. In our study such a rate was reached in only one period, which makes us think that under notification must be fairly large. On the other hand, environmental factors such as temperature, atmospheric pollution, and relative humidity could have a certain bearing on influenza activity and mortality. ${ }^{31-34}$ This type of factor was not considered in the present study and could perhaps go some way towards explaining, at least partly, the excesses of mortality detected.

Finally, another datum that reinforces the value of the mortality indicator in improving the detection of influenza epidemics in our study is the fact that mortality has increased before morbidity, except for the 1989-90 season in which there was intense influenza activity in both Europe and throughout Spain. ${ }^{9}$ This fact could be explained in two ways: (1) The indicator that best predicts large scale epidemic activity is reported morbidity. Mortality would then be considered as a complementary indicator. In our series the increase in reported morbidity preceded the increase in mortality only in the 1989-90 season. It should therefore be acknowledged that there was no epidemic in the other four seasons, but in two seasons there was also epidemic activity which was identified by virus isolements and by other indicators. (2) The indicator that best predicts epidemic activity in our milieu is general mortality. If it is a question of a surveillance system being able to detect a health problem, and 
moreover of doing so early, then it is clear that in all the epidemic seasons under study the mortality indicator enabled us to detect influenza epidemics. In addition, mortality began to increase very early in two of the epidemics. This, in our opinion, is the best explanation.

Again, when studying the influence of one model on another it can be seen that morbidity is influenced by the mortality registered in the four previous weeks, while the mortality series does not seem to be affected by previously reported cases of influenza-like illness. $\mathrm{Ob}$ viously, this correlation is not causal. It can therefore be affirmed not only that mortality is a good indicator of influenza activity in our milieu, but moreover that it is independent of notified morbidity.

The disadvantage of using the indicator based on mortality, it has been argued, is the delay in the availability. In our case, this limitation was not observed as the number of deaths occurring on the previous day is available daily in the towns studied.

Once it is assumed that mortality is a valid criterion and that the model based on early observation of deaths is useful, it would be interesting, as Sprenger et $a^{29}$ point out, to be able to determine from what level of increase measures ought to be adopted. Some authors $^{3536}$ define an epidemic as occurring when the observed value exceeds the expected value by more than two SDs for two consecutive weeks.

In our study covering five seasons, there was epidemic activity only in three, and mortality indicator was increased in the above mentioned way in all of them. In the two seasons without influenza epidemic activity, the mortality indicator did not show any significant increase. However, because this study involved only a small number of seasons and there is not an absolute standard that enables us to define the beginning of an influenza epidemic, ${ }^{37} 38$ the final decision on declaring an epidemic and instituting control measures, ${ }^{39} 40$ should be made by evaluating all the indicators included in the influenza surveillance programme.

\section{Appendix}

THE PROPOSED MODEL FOR THE MORTALITY SERIES WAS:

$$
\begin{gathered}
\left(1-f_{1} B-f_{2} B^{2}\right)\left(y_{t}-\bar{y}\right)=e_{t} \\
f_{1}=0.429 \\
f_{2}=0.272
\end{gathered}
$$

OR THE EQUIVALENT FORM:

$$
y_{t}-\bar{y}=\frac{1}{\left(1-f_{1} B-f_{2} B^{2}\right)} e_{l t}
$$

Once the analysis of intervention had been completed, the model was expressed as

$$
\begin{gathered}
y_{t}-\bar{y}=d_{a} D_{a}+d_{b} D_{b}+d_{c} D_{c}+d_{d} D_{d}+ \\
+\frac{1}{\left(1-f_{1} B-f_{2} B^{2}\right)} e_{l t}
\end{gathered}
$$

Where:

$D=$ intervention carried out in the mortality series and

$d=$ parameter of intervention

$D a, D b, D c, D d$, dummy variables which take the value of 1 for weeks shown in table 1 and 0 in the other cases.

$$
\begin{aligned}
& \bar{y}=153.646 \\
& d_{a}=39.62 \\
& d_{b}=33.49 \\
& d_{c}=25.56 \\
& d_{d}=25.83
\end{aligned}
$$

THE PROPOSED MODEL FOR THE MORBIDITY SERIES IS

$$
\begin{gathered}
\left(1-g_{1} B-g_{2} B^{2}-g_{3} B^{3}\right)\left(s_{t}-\vec{s}\right)=e_{2 t} \\
g_{1}=0.164 \\
g_{2}=0.191 \\
g_{3}=0.490
\end{gathered}
$$

or the equivalent form

$$
s_{t}-\bar{s}=\frac{1}{\left(1-g_{1} B-g_{2} B^{2}-g_{3} B^{3}\right)} e_{2 t}
$$

Once the analysis of intervention was completed, the model is expressed as

$$
\begin{gathered}
s_{t}-\bar{s}=w_{a} G_{a}+w_{b} G_{b}+w_{c} G_{c}+ \\
+\frac{1}{\left(1-g_{1} B-g_{2} B^{2}-g_{3} B^{3}\right)} e_{2 t}
\end{gathered}
$$

Where:

$G=$ intervention carried out in the morbidity series

$w=$ intervention parameter

$G_{a}, G_{b}, G_{c}, G_{d}$, dummy variables which take the value of 1 for weeks shown in Table 2 and 0 in other cases.

$$
\begin{aligned}
& \bar{s}=474 \cdot 95 \\
& w_{a}=1604 \cdot 575 \\
& w_{b}=4409 \cdot 780 \\
& w_{c}=1863 \cdot 026
\end{aligned}
$$

Finally, we fit the following autoregressive model to the mean corrected bivariate time series 
$\left\{y_{t}^{*}\right\}$, mortality series and $\left\{s_{t}^{*}\right\}$ morbidity series:

$$
\begin{aligned}
{\left[\begin{array}{c}
y_{t}^{*} \\
s_{t}^{*}
\end{array}\right] } & =\left[\begin{array}{cc}
0.313 & 0 \\
1.639 & 1 \cdot 141
\end{array}\right]\left[\begin{array}{l}
y_{t-1}^{*} \\
s_{t-1}^{*}
\end{array}\right]+ \\
+ & {\left[\begin{array}{cc}
0 \cdot 19 & 0 \\
2 \cdot 388 & -0 \cdot 397
\end{array}\right]\left[\begin{array}{l}
y_{t-2}^{*} \\
s_{t-2}^{*}
\end{array}\right]+} \\
& +\left[\begin{array}{cc}
0 & 0 \\
-0.615 & 0
\end{array}\right]\left[\begin{array}{l}
y_{t-3}^{*} \\
s_{t-3}^{*}
\end{array}\right]+ \\
+ & {\left[\begin{array}{cc}
0 & 0 \\
-1 \cdot 529 & 0
\end{array}\right] \cdot\left[\begin{array}{l}
y_{t-4}^{*} \\
s_{t-4}^{*}
\end{array}\right]+\left[\begin{array}{l}
e_{3 t} \\
e_{4 t}
\end{array}\right] }
\end{aligned}
$$

Operating with this expression, we arrive at the relations between the mortality mean corrected series $\left\{y_{t}^{*}\right\}$ and the morbidity mean corrected series $\left\{s_{t}^{*}\right\}$

$$
\begin{gathered}
y_{t}^{*}=0.313 y_{t-1}^{*}+0 \cdot 19 y_{t-2}^{*}+e_{3 t} \\
s_{t}^{*}+1.639 y_{t-1}^{*}+1 \cdot 141 s_{t-1}^{*}+2 \cdot 388 y_{t-2}^{*}- \\
-0.397 s_{t-2}^{*}-0.615 \mathrm{y}_{t-3}^{*}-1.529 \mathrm{y}_{t-4}^{*}+e_{4 t}
\end{gathered}
$$

or equivalently for the last second equation

$$
\begin{gathered}
s_{t}^{*}=\frac{\left(1 \cdot 639+2 \cdot 388 B-0 \cdot 615 B^{2}-1 \cdot 529 B^{3}\right) B}{\left(1-1 \cdot 141 B+0 \cdot 397 B^{2}\right)} y_{t}^{*}+ \\
+\frac{1}{\left(1-1 \cdot 141 B+0 \cdot 397 B^{2}\right)} e_{4 t}
\end{gathered}
$$

We thank Professor M J Campbell for his critical reading of the manuscript, Pilar Reguera and Gloria Carmona for obtaining the study data and the staff of the funeral services for their kind collaboration.

1 Langmuir AD. The surveillance of communicable diseases of national importance. $N$ Engl f Med 1963;268:182-92.

2 Thacker SB, Berkelman RL. Public health surveillance in the United States. Epidemiological Rev 1988;10:164-90.

3 Declich S, Carter AO. Public health surveillance: historical origins, methods and evaluation. Bul WHO 1994;72:285304 .

4 Istre GR. Disease surveillance at the state and local levels. In: Halperins W, Baker EL eds. Public health surveillance. New York: Van Nostrand Reinhold, 1992;42-55.

5 Vidal Tort J. ¿Cómo viajan los virus de la gripe? Med Clin (Barc) 1994;102:418-19.

6 Cabezas JA, Hannoun C. La gripe y sus virus. Investigación y Ciencia 1990;159:63-9.

7 Ghendon Y. Influenza surveillance-update. Bull WHO 1991; 69:509-15.

8 In: WHO/Geigy. Consultation of the standardization and improvement of influenza surveillance, Monaco Septembre 1991.

9 Pérez-Breña, de Miguel C, Ordobas M, et al. Sistema de vigilancia para el estudio de la circulación gripal en Madrid durante el quinquenio 1986-1991. Med Clin (Barc) 1994; 102:401-6.

10 Borrell Thió C, Plasencia Taradach A, Thió Fernández de Henestrosa S, Marti-Recober M. Vigilància epidemiologica de la mortalitat a partir dels enterraments. Gac Sanit 1991;5:6-16.
11 Statistics Institute of Catalonia. Statistics annual of Catalonia, 1991. Barcelona: Generalitat de Catalunya, 1992.

12 Department of Health and Social Security. Resolution of 7 of December of 1981 establishing the procedure of notifying statutory diseases. Diari oficial de la generalitat 1982; Janstatutory diseases.

13 Box GEP, Jenkins GN: Time series analysis: forecasting and control. San Francisco: Holden-Day, 1976.

14 Choi K, Thacker S. An evaluation of influenza mortality surveillance, 1962-1979. Am $\mathcal{F}$ Epidemiol 1981;113:22735.

15 Choi K, Thacker S. An evaluation of influenza mortality surveillance, 1962-1979. I. Time series forecast of expected pneumonia and influenza deaths. Am $\mathcal{F}$ Epidemiol 1981;113:215-26.

16 Brockwell PJ, Davis RA: Time series: theory and methods. Berlin: Ed. Springer-Verlag, 1991.

17 Tiao, GG. An introduction to multiple time series analysis. Med Care 1993;31:4571-4.

18 Stroup DF, Thacker SB, Hendon JL. Application of multiple time series analysis to the estimation of pneumonia and influenza mortality by age 1962-1983. Stat Med 1988;7: influenza $1045-59$.

19 Fleming DM, Cross KW. Respiratory syncytial virus or influenza? Lancet 1993;342:1507-10.

20 Sprenger MJW, Mulder PGH, Beyer WE, Strik RV, Masure W. Impact of influenza on mortality in relation to age and underlying disease. 1967-1989. Int $\mathcal{F}$ Epidemiol 1993;22: 334-40.

21 Lui Kung-Jong, Kendal AP. Impact of influenza epidemics on mortality in the United States from October 1972 to May 1987. Am F Public Health 1987;77:712-15.

22 Glezen WP. Serious morbidity and mortality associated with influenza epidemics. Epidemiol Rev 1982;4:25-44.

23 PHLS Communicable Disease Surveillance Centre. In fluenza surveillance. Communicable Disease Report 1992;2: 1 .

24 Joseph CA, Dedman D, Fern K, Chakraverty P, Watson JM. Influenza surveillance in England and Wales: November 1991 - June 1992. Communicable Disease Report 1992;2:R149-R150.

25 Sullivan KM, Monto AS, Longiwi IM. Estimates of the US Health impact of influenza. Am $\mathcal{F}$ Public Health 1993;83: 1712-16.

26 Monto AS, Sullivan KM. Acute respiratory illness in the community. Frequency of illness and the agents involved. Epidemiol Infect 1993;110:145-60.

27 Connolly AM, Saldon RL, Lervy B, Williams DH. What are the complications of influenza and can they be prevented? Experience from the 1989 epidemic of $\mathrm{H} 3 \mathrm{~N} 2$ influenza $\mathrm{A}$ in general practice. BMf 1993;306:1452-4.

28 Palmer SR, Salmon RL, Smith RMM. Influenza surveillance Wales 1988-1989. MMWR 1990;39:913-14.

29 Sprenger MJW, Mulder PGH, Beyer WEP, Masurel N. Influenza: relation of mortality to morbidity parameters Netherlands 1970-1989. Int f Epidemiol 1991;20:1118-24.

30 Fleming DM, and Ayres JG. Diagnosis and patterns of incidence of influenza-like illness and the common cold in general practice $f R$ Coll Gen Pract 1988;38:159-62.

31 Hope-Simpson RE. The role of season in the epidemiology of influenza. $\mathcal{F}$ Hyg Camb 1981;86:35-47.

32 Kunst AE, Looman CWN, Mackenbach JP. Outdoor air temperature and mortality in the Netherlands: A timeseries analysis. Am f Epidemiol 1993;137:331-41.

33 Rao BL, Baneriee K. Influenza surveillance in Pune, India 1978-1990. Bull WHO 1993;71:177-81.

34 Romieu I, Cortes Lugo M, Ruiz Velasco S, Sánchez S, Meneses F, Hernández $M$. Air pollution and school absenteeism among children in Mexico city. Am $\mathcal{F}$ Epidemio 1992;136:1524-31.

35 Costagliola D, Flahault A, Galinec D, et al. A routine too for detection and assessment of epidemics of influenza-
like syndromes in France. Am 7 Public Health $1991 ; 81$ : like synd
lit-9.

36 Valleron AJ, Carra TF, Garnerin PH. Early detection of epidemic influenza. Lancet 1992;339:57-8

37 Snaken R, Lion J, Casteren VV, et al. Five years of sentinel surveillance of acute respiratory infections (1985-90). The benefits of an influenza early warning system. Eur $\mathcal{F}$ Epidemiol 1992;8:485-90.

38 Dab W, Quenel P, Cohen JM, Hannoun C. A new influenza surveillance system in France: the ille de France "Grog". 2. Validity of Indicators (1984-89) Eur f Epidemiol 1991; 7:579-87.

39 Wiselka $M$. Influenza: diagnosis, management and prophylaxis. BMF 1994;308:1341-45.

40 Serwint J. Pediatrician-dependent barriers in influenza vaccine administration. Ped Infect Dis $¥$ 1993;12:956-68. 